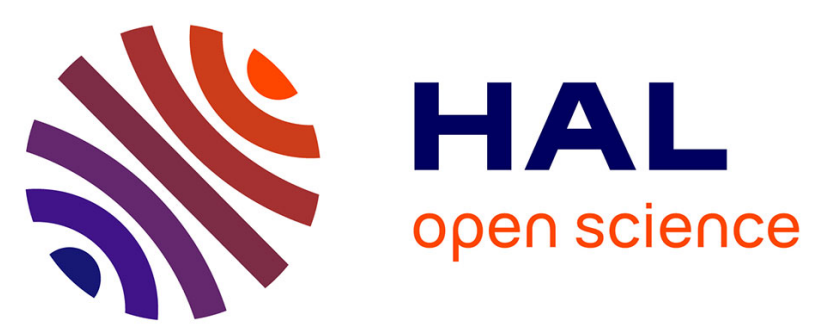

\title{
L'accueil des Jeux du Sud-Est asiatique à Vientiane: un révélateur des modes de production urbaine au coup par coup
}

Clément Musil, Karine Peyronnie, Bounleuam Sisoulath

\section{To cite this version:}

Clément Musil, Karine Peyronnie, Bounleuam Sisoulath. L'accueil des Jeux du Sud-Est asiatique à Vientiane: un révélateur des modes de production urbaine au coup par coup. Franck E. (dir.); Sanjuan T .(dir.). Les territoires de l'urbain en Asie: une nouvelle modernité ?, CNRS Editions, p.151-170, 2015, 978-2-271-09004-1. ird-01343413

\section{HAL Id: ird-01343413 \\ https://hal.ird.fr/ird-01343413}

Submitted on 11 Jul 2016

HAL is a multi-disciplinary open access archive for the deposit and dissemination of scientific research documents, whether they are published or not. The documents may come from teaching and research institutions in France or abroad, or from public or private research centers.
L'archive ouverte pluridisciplinaire HAL, est destinée au dépôt et à la diffusion de documents scientifiques de niveau recherche, publiés ou non, émanant des établissements d'enseignement et de recherche français ou étrangers, des laboratoires publics ou privés. 


\title{
L'accueil des Jeux du Sud-Est asiatique à Vientiane : un révélateur des modes de production urbaine au coup par coup
}

\author{
Clément Musil, Karine Peyronnie \\ et Bounleuam Sisoulath
}

La capitale de la République démocratique populaire lao, Vientiane, a accueilli en décembre 2009 la 25e édition des Jeux du Sud-Est asiatique. L'organisation de cette compétition sportive régionale ${ }^{1}$ fut l'occasion pour Vientiane de lancer de vastes chantiers pour améliorer les équipements sportifs et urbains existants, mais également pour en construire de nouveaux.

L'organisation d'une manifestation sportive d'envergure internationale, souvent qualifiée de "méga-évènement» dans la littérature relative à l'urbanisme évènementiel, est en général pour la ville hôte l'occasion de réaliser de nouveaux équipements, de résoudre des problèmes d'urbanisme (problem solving) et de lancer des politiques d'aménagement. Certains travaux sur le sujet se sont attachés à décrypter des logiques d'action particulières telle que l'«urban regeneration » (Gratton et al., 2005 ; Coaffee, 2007). D'autres ont interrogé la rentabilité des équipements réalisés (équipements de transport, sportif et d'accueil) qui, après la tenue de l'évènement, ont vocation à être recyclés et valorisés (Suborg et al., 2008).

Pour les métropoles accueillant des évènements sportifs majeurs, tels que les Jeux olympiques ou les coupes du monde de football, les stratégies d'aménagement déployées reposent généralement sur deux axes : le premier vise à aménager, ou à réaménager, les zones où se tiennent les principales animations; le second porte sur l'amélioration des liaisons physiques entre les zones où se déroulent les manifestations et les principaux lieux de centralité des villes organisatrices (construction de routes ou d'équipements de transport collectif) (Gold et Gold, 2007). Ces opérations permettent en outre d'initier des montages techniques et

\footnotetext{
${ }^{1}$ Les Jeux du Sud-Est asiatique réunissent les pays membres de l'Association des nations de l'Asie du Sud-Est (Asean), c'est-à-dire le Laos, le Cambodge, la Malaysia, le Myanmar, Singapour, la Thaïlande, le Vietnam, Brunei, l'Indonésie, les Philippines, ainsi que le Timor-Leste, qui devrait devenir membre de l'Asean en 2015. Créée en 1959, cette manifestation se déroule tous les deux ans. Son organisation est confiée à l'un des pays membres de la fédération des Jeux du Sud-Est asiatique et le roulement de l'accueil de cette compétition suit l'ordre alphabétique des pays membres.
} 
financiers inédits liant des partenaires publics et privés (Andranovich et al., 2001). De ce point de vue, l'urbanisme évènementiel participe certes à l'établissement des conditions préalables de retombées positives (économique, visibilité et image de marque) pour la ville hôte, mais il concourt surtout à l'application de politiques nouvelles d'aménagement et à la réalisation de grands travaux (Gold et Gold, 2007).

Dans cette contribution, au regard de l'entrée choisie que constitue l'urbanisme évènementiel, les Jeux du Sud-Est asiatique de Vientiane apparaissent comme une expérience singulière, voire comme un cas limite. Par rapport aux autres métropoles de l'Asean qui organisent des évènements internationaux - souvent des villes "globales», connectées, et fortement peuplées comme Bangkok, Jakarta, Kuala Lumpur ou Singapour -, la capitale laotienne fait figure d'exception. Vientiane est en effet considérée comme la plus petite capitale d'Asie du Sud-Est continentale ${ }^{2}$ et, à l'échelle régionale, comme une ville de second rang, un lieu mineur de la métropolisation (Peyronnie, 2012), une ville dont la taille et les fonctions ne lui permettent pas de se placer dans le groupe des métropoles asiatiques dominantes (Goldblum et al., 2012). Néanmoins, les effets de l'internationalisation de l'économie urbaine à Vientiane sont visibles. Pour la réalisation de la plupart de ses projets urbains, la capitale du Laos est en situation de dépendance à l'égard des investissements étrangers, aussi bien sur la façon de concevoir les opérations que sur leur mise en œuvre ${ }^{3}$.

Au-delà de l'évènement sportif, l'accueil des Jeux du Sud-Est asiatique à Vientiane a pris un sens particulier tant sur le plan politique qu'économique. Pour le gouvernement laotien, l'organisation de ces Jeux était une occasion de «mettre le Laos sur la carte», d'exalter un sentiment national, de stimuler le potentiel touristique du pays et de valoriser l'image de la ville hôte qui organisait pour la première fois ces jeux ${ }^{4}$ (Creak, 2011). L'accueil de cette compétition a aussi permis au Laos de poursuivre sa dynamique d'intégration régionale engagée depuis le milieu des années 1980, en renforçant sa présence sur la scène politique et en assumant son statut de pays membre de l'Asean (Shrestha et Chongvilaivan, 2013) ${ }^{5}$. Toutefois, en raison d'un manque patent de capitaux, la préparation de cette compétition a conduit le gouvernement laotien à solliciter ses partenaires régionaux pour qu'ils financent et réalisent les équipements (sportifs et de transport) nécessaires au bon déroulement de la manifestation.

À partir de l'analyse du jeu des acteurs et contributeurs étrangers à la construction et à l'amélioration des équipements de la capitale laotienne, nous

\footnotetext{
${ }^{2}$ D'après le schéma directeur réalisé par l'Agence de coopération japonaise (Jica), le territoire administratif de la capitale de Vientiane comptait environ 800000 habitants en 2009. Cependant, les limites retenues englobent 9 districts (dont 4 urbains), eux-mêmes formés de 496 villages urbains et ruraux. La population urbaine était de l'ordre de 400000 habitants à la fin des années 2000.

3 Voir sur ce point la quatrième partie de l'ouvrage de Clément Charpentier et al. (2010).

${ }^{4}$ Si le Laos avait refusé d'organiser cet évènement, comme il l'avait fait en 1965, il aurait dû attendre le tour suivant, soit environ près de vingt ans, avant de pouvoir à nouveau l'organiser.

${ }^{5}$ À l'instar du Doi Moi vietnamien, le gouvernement laotien a adopté en 1986 un « nouveau mécanisme économique » visant à réduire l'intervention étatique dans le secteur économique. Engagé sur la voie de l'ouverture économique et diplomatique, le Laos est depuis membre d'organisations et de programmes de coopération régionale : Région du Grand Mékong (1992) et Asean (1997).
} 
montrons que la logique urbanistique accompagnant la programmation de ces opérations a tout simplement été absente à Vientiane. L'organisation de cette manifestation a avant tout eu vocation à renforcer l'image de la ville, et à entretenir les relations économiques et politiques entre le Laos et ses partenaires régionaux, plutôt qu'à résoudre des problèmes d'articulation des fonctions urbaines. Bien que l'organisation des Jeux du Sud-Est asiatique à Vientiane n'ait pas entraîné les effets urbanistiques escomptés, les contreparties scellées entre le gouvernement laotien et ses partenaires régionaux pour la réalisation des équipements ont, quant à elles, des impacts notoires sur la production et le réagencement des fonctions urbaines.

\section{L'organisation des Jeux du Sud-Est asiatique et le déploiement de logiques urbanistiques}

Les premiers Jeux du Sud-Est asiatique se sont tenus à Bangkok en 1959. Apparue dans un contexte de décolonisation, cette compétition avait pour objectif de promouvoir la fraternité et la paix dans la région, mais également de participer à l'émancipation politique des pays d'Asie du Sud-Est. Au début des années 1950, la création d'une autre compétition, les Jeux asiatiques, avait été perçue comme une défiance des pays du tiers-monde à l'égard des doctrines de supériorité occidentales et à l'égard de toutes formes de racisme et d'impérialisme (Hong, 2005 : 517). L'importance du sport dans les relations politiques a été poussée à son paroxysme avec l'organisation en 1963 des Ganefo (Games of the new emerging forces) à Jakarta et des Ganefo asiatiques en 1966 à Phnom Penh' .

Mis à part les enjeux politiques et diplomatiques, ces compétitions régionales comportaient des enjeux économiques. Elles constituaient de véritables occasions d'investissements pour les pays organisateurs. Ces derniers ont souvent lancé de vastes opérations visant à construire de nouveaux équipements (sportifs, hôteliers, de transport), à moderniser les centres des villes d'accueil ou encore à planifier l'aménagement de nouveaux quartiers. En raison de leur dimension régionale, et à la différence des méga-évènements, les Jeux du Sud-Est asiatique ne donnent pas lieu pour les villes hôtes à des transformations mobilisant d'importants volumes financiers. Néanmoins, des projets ponctuels sont engagés et certains sont porteurs de réelles innovations urbanistiques. Rétrospectivement, l'organisation des grandes compétitions sportives en Asie du Sud-Est est à ce sujet riche d'enseignements. Quelques exemples sont particulièrement significatifs.

En vue de l'accueil des Jeux asiatiques de 1962, puis des Ganefo l'année

\footnotetext{
${ }^{6}$ Initiés par le président indonésien Sukarno, les Ganefo s'inscrivent dans le sillage de la conférence des pays "non-alignés » de Bandung en 1955. Les Ganefo de Jakarta ont réuni des pays asiatiques, africains et sud-américains, ainsi que des pays socialistes. Cette compétition est apparue comme le produit d'une époque dans laquelle les pays du tiers-monde ont contesté l'esprit et la structure du mouvement sportif international, qui était alors contrôlé par les «puissances occidentales impérialistes». La tenue des Ganefo a prouvé que le sport constituait une ressource politique et diplomatique précieuse. Malgré tout, cette compétition, tout comme son pendant régional asiatique, n'a connue qu'une seule édition (Lutan et Hong, 2005).
} 
suivante, le Président indonésien Ahmed Sukarno souhaitait transformer Jakarta en une capitale asiatique modèle. Ce dernier a usé de ses relations et obtenu de l'Union soviétique un prêt de plus de 12,5 millions de dollars étatsuniens pour la réalisation d'un complexe sportif de 300 ha et d'un grand stade, le Bung Karno Stadium. Ces réalisations ont été accompagnées par la construction d'autres équipements - un hôtel de standing international, l'Indonesia Hotel, un centre commercial, le Sarinah, et des voies routières et autoroutières (Lutan, 2005: 419). En outre, l'académie d'architecture paysagère de Jakarta a été créée à cette occasion (Arifin, 2012).

Dans un autre registre, le complexe sportif du stade olympique de Phnom Penh constitue un exemple intéressant d'innovation architecturale et urbanistique suscité par les compétitions sportives régionales. Conçu par l'architecte Vann Molyvann, en collaboration avec l'architecte Vladimir Bodiansky et l'urbaniste Gérald Hanning, experts de l'ONU, ce stade avait été réalisé en vue d'accueillir les Jeux de l'Asie du Sud-Est péninsulaire ${ }^{7}$ de 1963, mais ces derniers ont été annulés en raison de la situation politique dans le pays. Le stade a finalement été utilisé pour les Ganefo asiatiques. Outre la réalisation du complexe sportif, qui constitue désormais un élément significatif de morphologie architecturale de la capitale cambodgienne, d'autres édifices furent construits, tel le marché olympique (marché russe).

L'organisation de tels Jeux a aussi été l'occasion d'introduire de nouvelles typologies architecturales. Par exemple, l'apparition du modèle des lotissements pavillonnaires à Bangkok (muban chatsan) est apparue lors de la première édition des Jeux asiatiques. "Le pavillonnaire s'est trouvé à la convergence des idéalités résidentielles modernes et de la recherche de nouveaux signes de distinction de la part des catégories moyennes émergentes » (Goldblum, 2000 : 60-61).

Plus récemment, en 2003, lors de l'accueil des 22 Jeux du Sud-Est asiatique par le Vietnam, la capitale du pays, Hanoi, a été dotée d'un nouveau complexe sportif (My Dinh) et d'un réseau de voies permettant d'y accéder et de circuler d'un équipement à l'autre (Lê Duc Tho, Lê Quang Dao, Mê Tri). Depuis, la nouvelle trame viaire a favorisé le développement de projets immobiliers (tours d'habitation, lotissements de villas) et permis de forger un nouveau pôle de centralité urbaine. Mis à part la réalisation d'équipements sportifs et de transport, les autorités ont imposé à Hanoi et à Hô Chi Minh-Ville (seconde ville hôte de ces Jeux) une campagne de nettoyage des rues ${ }^{8}$.

Parmi ces caractéristiques générales relatives à la préparation des compétitions sportives dans les villes d'Asie du Sud-Est, il apparaît que l'urbanisme qui y est associé dépasse le simple cadre de l'évènement pour atteindre la dimension d'un projet urbain d'envergure. Il existe ainsi un «rapport étroit entre l'évènement dans sa dimension urbanistique et sa mise en récit, qui lui donne une dimension temporelle excédant le temps de l'éphémère » (Gravari-Barbas et Jacquot, 2007 : 9). L'évènement urbain est conçu pour marquer l'agencement de la ville dans la durée

\footnotetext{
${ }^{7}$ Dénomination des Jeux du Sud-Est asiatique utilisée entre 1959-1977, avant que l'Indonésie et les Philippines ne rejoignent la compétition.

${ }^{8}$ Cette campagne visait notamment à dissimuler aux yeux des visiteurs et des investisseurs étrangers toutes formes de pratiques de commerce informel de rue pouvant nuire à l'image des villes du pays (Harms, 2003).
} 
en provoquant une réorganisation du tissu urbain et en accordant un surcroît de légitimité à certains projets.

Les effets attendus de l'urbanisme évènementiel, à savoir la construction d'équipements, leur intégration au tissu urbain existant, les stratégies élaborées concernant « l'après » évènement, le recyclage des infrastructures, mais également la rentabilité des investissements, sont autant de composantes à prendre en compte dans la programmation de projets lancés à l'occasion de manifestations internationales majeures. Dans le cas de Vientiane, la mise aux normes des équipements existants et la construction de nouveaux équipements constituaient un passage obligé, au même titre que pour les autres villes ayant accueilli les éditions précédentes des Jeux du Sud-Est asiatique. Mais à la différence des épisodes pionniers, les travaux engagés à Vientiane ont avant tout constitué des occasions commerciales pour les investisseurs intervenant dans le sillage des pays partenaires de la République démocratique populaire lao sans réelle recherche d'une production urbaine adossée à un projet de ville.

\section{La préparation des Jeux de Vientiane : des opérations d'aménagement tributaires d'une aide régionale et d'investissements étrangers}

Avant l'accueil des Jeux, Vientiane disposait d'un nombre restreint d'infrastructures sportives : un ancien stade de taille modeste situé dans le centre de la capitale ou encore deux terrains de golf ne répondant pas aux standards internationaux. Le Lao-International Trade Exhibition and Convention Center, construit à l'occasion d'un sommet de l'Asean en 2004, a toutefois été réutilisé lors des Jeux. La capitale du Laos manquait également d'équipements de télécommunications pour assurer les retransmissions télévisées à l'étranger et sur Internet, et de routes de qualité permettant le déplacement des délégations et des visiteurs.

Outre le secteur hôtelier, qui a bénéficié d'investissements privés, le Laos a pu compter sur le soutien financier et technique de la Chine, du Vietnam, de la Thaïlande, du Japon, de la Corée du Sud et d'autres pays de la région pour construire et améliorer les équipements sportifs, les routes et les infrastructures de télécommunications. Si le soutien extérieur dont a bénéficié le Laos a été apporté par certains des membres de la fédération des Jeux du Sud-Est Asiatique, tels que la Thaïlande et le Vietnam, ce sont en fait les principaux partenaires économiques du Laos qui, à la demande du gouvernement laotien, ont participé au financement des équipements ${ }^{9}$. Selon les informations diffusées par la presse, le montant global des investissements pour la construction des équipements sportifs et l'amélioration du réseau routier aurait été approximativement de 210 millions de dollars étatsuniens.

\footnotetext{
${ }^{9}$ En 2009, près des 3/4 des investissements étrangers en République démocratique populaire lao provenaient de la Thaïlande (27\%), de la Chine $(23 \%)$ et du Vietnam (21\%). Les flux d'investissements d'origines japonaise et coréenne ne représentaient, quant à eux, que $4 \%$ et $5 \%$. Ces investissements sont principalement dirigés vers la construction et l'exploitation de barrage hydroélectrique, et vers l'extraction de ressources minières (Banque mondiale, 2009 : 18).
} 
Deux aspects relatifs à la préparation des Jeux présentent un intérêt réel : la production des équipements sportifs d'une part et celle des équipements de transport d'autre part. Le recensement des différentes opérations menées permet de cerner les spécificités de la dimension internationale de la production des infrastructures urbaines dans la capitale (fig. 1).

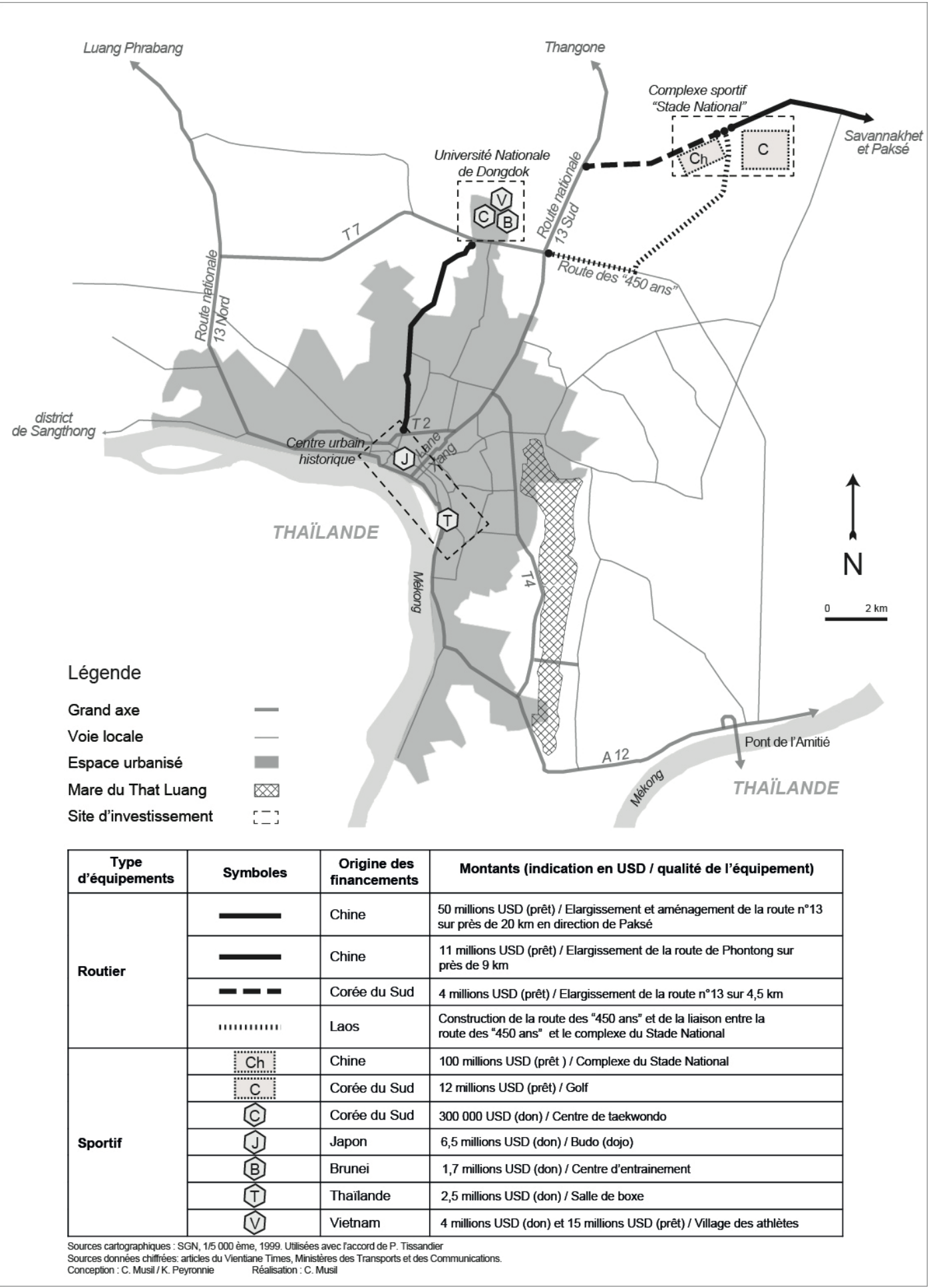

Figure 1 : Origine des principaux investissements mobilisés pour la réalisation des équipements des Jeux du Sud-Est asiatique de Vientiane en 2009. 
Équipements sportifs et maîtrise d'œuvre internationale

En vue du déroulement des épreuves sportives, plusieurs équipements répartis sur trois sites (le complexe du Stade national, l'Université de Dongkok et le centre historique) ont été construits. Ces réalisations, dont les financements ont été apportés par plusieurs pays participants ou non à la compétition, témoignent d'une solidarité régionale, d'enjeux diplomatiques et d'arrangements commerciaux.

Pour construire le complexe du Stade national - comprenant entre autres une piscine, une salle omnisports et un stade de 25000 places-, un consortium d'entreprises chinoises, coordonnées par la Suzhou Industrial Park Overseas Investment Company, a été sollicité en 2007. Pour procéder à la réalisation de cet équipement, le gouvernement laotien a bénéficié d'un prêt d'un montant approximatif de 100 millions de dollars étatsuniens accordé par la China Development Bank ${ }^{10}$. Les négociations entre le gouvernement laotien et la banque se sont déroulées dans le secret (Stuart-Fox, 2009 : 142). Dans les termes de l'échange, la banque chinoise a par exemple imposé au gouvernement laotien de recourir aux services de la Yunnan Construction Engineering Group ${ }^{11}$ afin d'assurer la maîtrise d'œuvre. Aux abords du Stade national, la compagnie sud-coréenne Booyoung a également financé la réalisation d'un nouveau parcours de golf pour plus de 12 millions de dollars étatsuniens.

Dans le périmètre de l'Université nationale, en échange de l'obtention de la construction du parcours de golf, Booyoung a contribué sous forme de dons à la réalisation du centre de Taekwondo (300 000 dollars étatsuniens). Sur ce second site, le groupe Hoang Anh Gia Lai apparaît comme l'investisseur le plus important. Intervenant dans le sillage de la diplomatie vietnamienne, ce groupe a financé la construction du village des athlètes (19 millions de dollars étatsuniens). Davantage connu pour son activité dans l'industrie forestière au Vietnam comme au Laos, Hoang Anh Gia Lai s'est spécialisé dans la promotion immobilière et la construction depuis le milieu des années 2000 (Kenney-Lazar, 2010). Les Jeux ont ainsi permis à cet investisseur de prendre position sur le marché immobilier de Vientiane.

Les installations sportives situées dans le centre de la capitale ont été financées sous forme de dons. Pour un montant de 2,5 millions de dollars étatsuniens, la Thaïlande a financé la rénovation du stade existant et la construction d'une salle de boxe. Le Japon, pourtant extérieur à la fédération des Jeux du Sud-Est asiatique, a pour sa part contribué à la réalisation d'un dojo (centre d'arts martiaux), en accordant un don de 6,5 millions de dollars étatsuniens. Ces deux derniers exemples mettent en évidence des logiques moins commerciales que politiques visant surtout à consolider les relations bilatérales et l'influence des pays donateurs en République démocratique populaire lao par l'expression d'une solidarité régionale.

\footnotetext{
${ }^{10}$ Avec l'Eximbank et l'Agricultural Bank of China, la China Development Bank est directement contrôlée par le gouvernement chinois. D’après la presse laotienne, le président de la China Development Bank est un personnage important ayant rang de ministre.

11 Yunnan Construction Engineering Group, http://www.yncegc.com.cn/, consulté le 14.08.13.
} 
L'organisation des Jeux aurait pu être considérée par les autorités comme une occasion de produire une centralité, faisant jusqu'alors défaut dans la capitale. Mais la distribution à l'échelle de la ville des équipements sportifs ne permet pas de penser qu'une stratégie particulière ait été élaborée. Les épreuves sportives se sont en effet déroulées sur trois sites éloignés les uns des autres: le Stade national et l'Université nationale sont respectivement situés à 16 et $9 \mathrm{~km}$ du centre historique. Si l'accueil de l'évènement sportif a bien produit de nouvelles polarités, notamment en périphérie nord-est de la ville, avec le renforcement du pôle universitaire, il n'a en revanche pas suscité une nouvelle échelle de centralité pour structurer le développement urbain, ni même entraîné la construction de nouveaux quartiers d'habitation ou d'activités. L'effet de l'accueil des Jeux sur l'organisation de la ville paraît à ce titre limité.

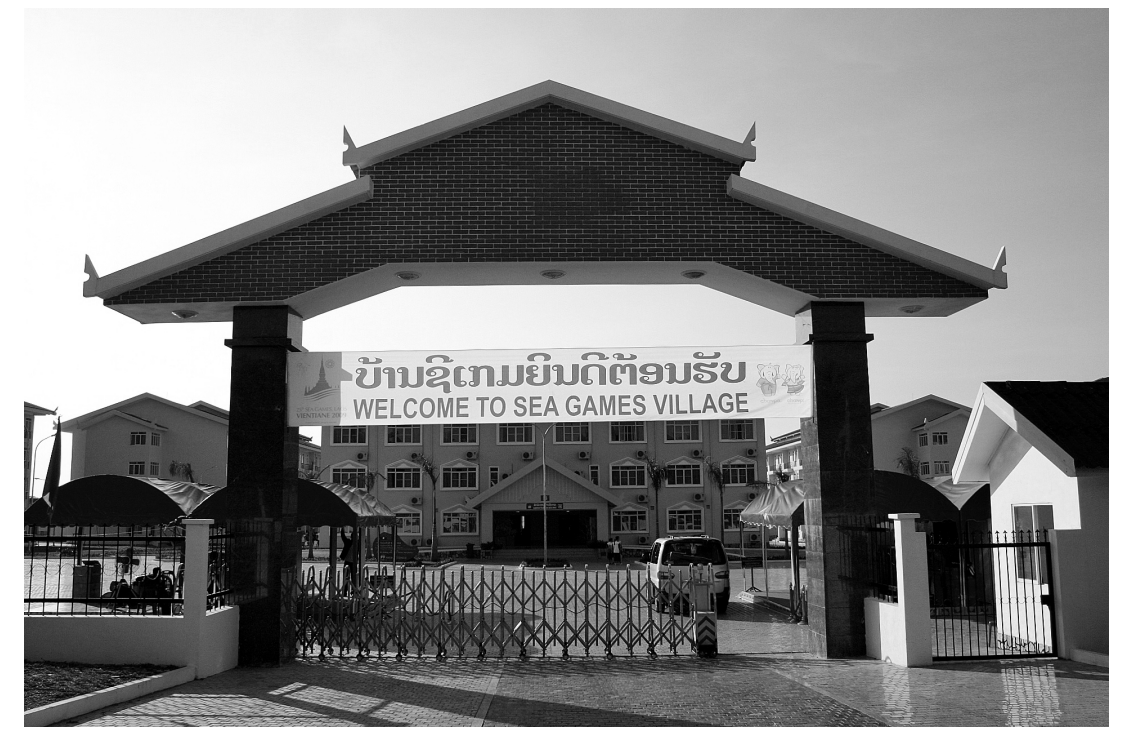

Figure 2 : L'entrée principale du village des athlètes financé par le Vietnam (Source : Clément Musil, 2009.)

Depuis la clôture des Jeux, la réutilisation des équipements sportifs est irrégulière et limitée. Le complexe du Stade national est utilisé occasionnellement pour des rassemblements populaires (célébrations nationales et activités politiques) et des rencontres sportives. Malgré l'organisation de ces manifestations, peu de recettes sont dégagées. Par conséquent, en raison d'un manque de budget affecté à leur maintenance, certains équipements, comme la piscine, présentaient quelques mois après leur mise en service des signes de dégradation. Le parcours de golf est en revanche entretenu et exploité par la société Booyoung. Enfin, les équipements sportifs du centre historique (dojo, salle de boxe), ceux situés sur le campus universitaire et le village des athlètes sont régulièrement utilisés. Reconverti en logements pour étudiants, ce dernier héberge désormais près de 800 étudiants de l'Université Nationale du Laos dont plus d'un-tiers d'étrangers en 201412.

12 Le prix annuel de la location par étudiant est d'environ 85 euros pour un étudiant laotien contre 215 euros pour un étudiant étranger. La recette des loyers est de l'ordre de 100000 euros par an. 
Dans la programmation de ces équipements, même pour les plus coûteux comme le Stade national, aucune stratégie d'amortissement des investissements effectués n'a été adoptée. Au-delà du bon déroulement de la compétition, la réalisation de ces équipements sportifs a surtout été guidée par des considérations commerciales et diplomatiques visant à financer l'évènement sans envisager formellement leur recyclage ni même les modalités de leur inscription dans la ville.

L'organisation des déplacements et les travaux de voirie, un bilan mitigé

Les investissements en matière d'équipements de transport constituent un second élément révélateur des logiques d'aménagement adoptées par les autorités à l'occasion des Jeux. En ce qui concerne les déplacements, l'objectif des pouvoirs publics était de s'assurer de la fluidité du trafic routier dans Vientiane pendant la durée de la manifestation. L'accent avait alors été mis sur l'amélioration du transport public, la création de nouvelles aires de stationnement automobiles et sur l'amélioration du réseau routier pour limiter la congestion routière et embellir certains axes (Lane Xang, l'Avenue Kaysone, la route nationale $\mathrm{n}^{\circ} 13$ ).

L'amélioration de l'offre en transport public n'a pas mobilisé une assistance extérieure spécifique. Toutefois, pour l'accueil des Jeux, les autorités ont fait preuve d'initiatives dans ce domaine. Elles ont créé quatre nouvelles lignes de bus publics, élevant ainsi leur nombre à 14 en 2009 et amélioré l'information aux voyageurs aux arrêts de bus (affichage des horaires et de la carte du réseau). Vientiane a ensuite innové en mettant en service une dizaine de minibus électriques achetés à un constructeur chinois (pour un montant d'environ 170000 dollars étatsuniens). Même si aucune infrastructure coûteuse de transport public n'a été réalisée, les Jeux ont permis aux autorités urbaines de tester une nouvelle organisation de son réseau de bus, sans pour autant recourir au soutien de partenaires étrangers.

Les actions de modernisation du réseau routier apparaissent au contraire comme des traits spécifiques de la dimension internationale de la production des infrastructures urbaines. À l'occasion des Jeux, deux types d'intervention ont été conduits : le percement et l'élargissement de voies. Concernant le premier type d'intervention, deux axes ont été percés. Le premier est une section de 3,5 km de la route des « 450 ans» de Vientiane - route qui constitue désormais une voie circulaire structurante de la ville, elle connecte la route nationale $\mathrm{n}^{\circ} 13$ au pont de l'Amitié reliant le Laos à la Thaïlande ${ }^{13}$. Le second est un axe de 6,5 km reliant la route des « 450 ans » au Stade national. Pour ces réalisations, les fonds mobilisés émanaient du gouvernement laotien, mais les autorités ont sollicité des investisseurs locaux pour compléter leur budget.

\footnotetext{
${ }^{13}$ Les célébrations des « 450 ans » de Vientiane se sont déroulées en décembre 2010, soit un an après les Jeux du Sud-Est asiatique. Les $21 \mathrm{~km}$ que compte la route circulaire des « 450 ans » étaient alors achevés.
} 


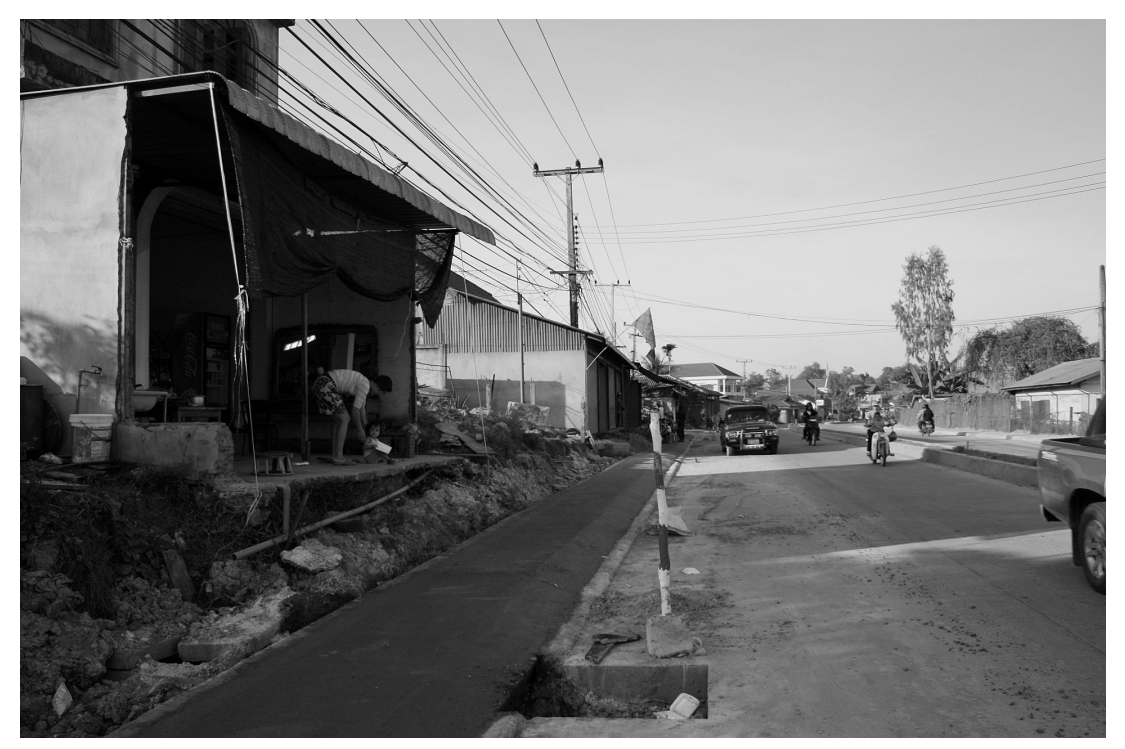

Figure 3 : Les travaux d'élargissement de la rue Phontong

(Source : Clément Musil 2009.)

Les opérations d'élargissement des routes ont, quant à elles, mobilisé de nombreux acteurs étrangers, tant pour leur financement que pour leur réalisation. À partir du Stade National, la route nationale $n^{\circ} 13$ en direction de Paksé a été élargie sur plus de $20 \mathrm{~km}$ pour un coût dépassant les 50 millions de dollars étatsuniens. Pour cette opération, le gouvernement laotien a obtenu un prêt de la banque d'État chinoise Eximbank. Le Yunnan Construction Engineering Group, déjà lié à la construction du Stade National, a effectué les travaux de voirie. L'élargissement d'une autre section de $4,5 \mathrm{~km}$ de la route nationale $\mathrm{n}^{\circ} 13$, à l'ouest du stade en direction du centre historique, a été financé par la Corée du Sud par un prêt de 4 millions de dollars étatsuniens. Cette opération a associé une compagnie de travaux publics sud-coréenne (Hanbaek-KCI) à un bureau de consultants sud-coréen (Isan), en partenariat avec un bureau local (Laos Transport Engineering Consultant). Une autre section routière de plus de $9 \mathrm{~km}$, l'axe Phontong reliant l'Université nationale au centre de la capitale, a été élargie par une compagnie de construction chinoise (Qin Huang Dao Tong Lian Group). Ce chantier, dont le coût était estimé à environ 11 millions de dollars étatsuniens, fut financé par le gouvernement laotien.

Certaines des opérations évoquées ont connu d'importants retards et n'étaient pas achevées pour le début des Jeux. Si la saison des pluies a ralenti les travaux, ce sont surtout les montages techniques et financiers faisant intervenir un grand nombre de prestataires locaux et étrangers qui ont été les principales sources de complication. Les autorités (Département des transports de la capitale de Vientiane, et ministère des Transports et des communications) ont rencontré des difficultés dans le suivi des projets. D'une opération à l'autre, la diversité des techniques de construction employées imposait aux maîtres d'ouvrages de disposer de compétences particulières pour contrôler les travaux. Ces différences de techniques ont au final eu un impact négatif sur la qualité des ouvrages : certaines sections routières se sont rapidement dégradées après la tenue des Jeux, en raison de l'utilisation de matériaux de construction de mauvaise qualité. 
Malgré une impression de cohérence dans l'articulation de ces projets routiers - qui sont en fait des équipements dont la réalisation était inscrite au schéma d'aménagement des transports de la capitale produit en 2008 par la coopération japonaise (Jica et al., 2008) -, la mise en œuvre de ces opérations révèle des carences et des dysfonctionnements dans la gestion des chantiers. Les actions portant sur l'amélioration du réseau routier de la capitale pointent: d'une part, le problème posé par la qualité des ouvrages, et dans ce sens remettent en question leur pérennité ; d'autre part, la réalisation de ces travaux souligne la dépendance récurrente de Vientiane à l'égard d'une aide extérieure pour le financement et la construction de ses infrastructures. La précipitation dans laquelle la ville s'est retrouvée à la veille des Jeux en raison du retard pris par chaque opération a en outre révélé des difficultés considérables dans la capacité des maîtres d'ouvrage à gérer leurs relations avec les maîtres d'œuvre.

Bien que les voies nouvellement créées et élargies améliorent l'accessibilité de Vientiane et participent à la structuration de son territoire, les autorités ne mettent pas en œuvre une stratégie pour mobiliser de manière continue des capitaux destinés au développement du réseau routier. Les modes de financement de ces équipements, à l'instar des équipements sportifs construits lors des Jeux, semblent reposer sur des arrangements au coup par coup impliquant des contreparties spécifiques.

\section{Les logiques d'acteurs et leurs effets sur la production des équipements urbains}

La caractéristique dominante de la production des équipements sportifs et routiers construits à l'occasion des Jeux de Vientiane repose sur l'utilisation de financements extérieurs, sous forme de dons et de prêts. Si la première source relève d'une solidarité régionale, elle est également l'expression d'une logique d'influence des pays donateurs. Par conséquent, pour l'utilisation des dons, les donateurs ont imposé aux autorités laotiennes des contraintes, notamment dans le choix des concepteurs de projets et des maîtres d'œuvre. La seconde source de financements, celle des prêts, implique des contreparties plus lourdes. Celles-ci apparaissent dans le cas de Vientiane comme des modalités nouvelles de production de la ville. Ces pratiques traduisent certaines dérives relatives à la logique de projet à laquelle ont recours les décideurs politiques laotiens pour la réalisation des équipements urbains.

\section{Conception et montage des opérations}

Les montages technico-financiers des opérations réalisées grâce à des prêts étrangers présentent deux particularités. La première est relative à la préparation des appels d'offres. Quelle que soit la nature des projets, équipements sportifs ou routiers, les créanciers conditionnent l'apport de leurs financements. Ils exigent en particulier l'association d'entreprises originaires de leur pays. Cette contrainte apparaît de manière directe avec la prescription d'un maître d'œuvre, comme dans 
le cas du Stade national et de l'élargissement de la route nationale $\mathrm{n}^{\circ} 13$ : le choix du Yunnan Construction Engineering Group a été effectué par les banques chinoises et non pas par des décideurs laotiens.

Une autre contrainte relative aux appels d'offres apparaît avec l'introduction de clauses restrictives. Dans le cas de la section routière financée par la Corée du Sud, le cahier des charges de cette opération prévoyait par exemple le recours à des techniques que seules les compagnies sud-coréennes pouvaient remplir, excluant de fait les entreprises locales. Les entreprises laotiennes n'ont toutefois pas été complètement écartées du dispositif, dans la mesure où elles ont joué un rôle en tant que sous-traitants, comme dans le cas de la route de Phontong financée par des capitaux chinois.

\section{De lourdes contreparties liées au financement des équipements}

La seconde particularité des opérations financées par des prêts étrangers renvoie aux contreparties que ces projets impliquent. Il apparaît que les investisseurs ayant financé un équipement sportif ou routier dans le cadre des Jeux ont obtenu comme mode de rétribution la mise à disposition de ressources naturelles; il s'agit en somme d'un échange de type « équipement contre ressource naturelle ». En réalité, les contreparties font l'objet d'intenses négociations, souvent dissimulées, entre les investisseurs et les autorités laotiennes. Par conséquent, ces contreparties peuvent à l'issue des négociations revêtir des natures différentes et prendre la forme de concessions foncières, forestières ou minières (tab. 1).

\begin{tabular}{|l|c|l|l|}
\hline \multicolumn{2}{|c|}{ Équipement sportif/routier } & $\begin{array}{c}\text { Contrepartie en ressource } \\
\text { naturelle }\end{array}$ \\
\hline $\begin{array}{l}\text { Opération } \\
\text { Localisation } \\
\text { (province) }\end{array}$ & $\begin{array}{c}\text { Montant de } \\
\text { l'investissement }\end{array}$ & \multicolumn{1}{c|}{$\begin{array}{c}\text { Origine de } \\
\text { l'investissement } \\
\text { Maître d'œuvre }\end{array}$} & $\begin{array}{c}\text { Nature de la concession / } \\
\text { durée ou surface }\end{array}$ \\
\hline $\begin{array}{l}\text { Route de } \\
\text { Phontong } \\
\text { Luang Namtha }\end{array}$ & 11 millions US\$ & $\begin{array}{l}\text { Chine } \\
\text { Qin Huang Dao Tong Lian } \\
\text { Group }\end{array}$ & $\begin{array}{l}\text { Concession } \\
\text { minière / 20 ans }\end{array}$ \\
\hline $\begin{array}{l}\text { Village des } \\
\text { athlètes } \\
\text { Attapeu }\end{array}$ & 19 millions US\$ & $\begin{array}{l}\text { Vietnam } \\
\text { Hoang Anh Gia Lai }\end{array}$ & $\begin{array}{l}\text { Concession } \\
\text { forestière / 10 000 ha }\end{array}$ \\
\hline $\begin{array}{l}\text { Complexe du } \\
\text { Stade national } \\
\text { Vientiane }\end{array}$ & 100 millions US\$ & $\begin{array}{l}\text { Chine } \\
\text { Yunnan Construction } \\
\text { Engineering Group }\end{array}$ & $\begin{array}{l}\text { Concession } \\
\text { foncière / 50 ans / 1000 ha }\end{array}$ \\
\hline $\begin{array}{l}\text { Golf } \\
\text { Vientiane }\end{array}$ & 12 millions US\$ & $\begin{array}{l}\text { Corée du Sud } \\
\text { Booyoung }\end{array}$ & $\begin{array}{l}\text { Concession de } \\
\text { l'équipement / 210 ha }\end{array}$ \\
\hline $\begin{array}{l}\text { Route des } \\
\text { « 450 ans » } \\
\text { Vientiane }\end{array}$ & 46 millions US\$ & $\begin{array}{l}\text { Gouvernement lao et } \\
\text { investisseurs d'origine } \\
\text { chinoise }\end{array}$ & $\begin{array}{l}\text { Parcelles foncières à } \\
\text { urbaniser }\end{array}$ \\
\hline
\end{tabular}

Tableau 1 : Le financement des Jeux du Sud-Est asiatique à Vientiane et ses contreparties.

Note: US\$ : dollars étatsuniens. Sources : Vientiane Times, 2009, «Phontong road paved for Sea Games », 16 novembre ; Vientiane Times, 2009, " Golf course ready for SEA Games to tee off, 21 août » ; Creak, Simon, 2009, «Little Laos awaits its big moment», Asia Times On Line, http://www.atimes.com/atimes/Southeast_Asia/KK05Ae01.html ; Kenney-Lazar, 2010, Land Concessions, Land Tenure, and Livelihood Change: Plantation Development in Attapeu Province, Southern Laos, Vientiane, Fulbright et the National University of Laos, 47 p. ; Musil, Clément, et Sèngdara Douangmyxay, 2010, " La question foncière à Vientiane, de la politique d'ouverture économique à l'ère des projets urbains ", dans Clément Charpentier, Sophie, Clément, Pierre, Goldblum, Charles, et Christian Taillard, dir., Vientiane, architectures d'une capitale: traces, formes, structures, projets, Paris, Cahiers de l'Ipraus, p. 383-402 
Ces échanges présentent des effets pernicieux pour le développement urbain et la gestion territoriale de Vientiane. D'abord, ce sont des arrangements léonins, qui désavantagent nettement la puissance publique. Après la livraison des équipements, les autorités ont à supporter les charges de leur maintenance et, dans le cas des équipements produits pour les Jeux, de leur recyclage. L'entretien du complexe du Stade national est par exemple devenu une charge supplémentaire pour le gouvernement. À l'opposé, les investisseurs ayant obtenu des concessions minières, forestières ou foncières en échange de la construction d'un équipement, vont durablement profiter des rendements d'exploitation.

Ensuite, par ces arrangements, la maîtrise du développement de la capitale laotienne échappe aux autorités urbaines. Du moins, elles ne paraissent plus en mesure de faire respecter les règlements d'urbanisme et les plans d'aménagement. L'illustration la plus significative de cette perte de contrôle reste le cas de la contrepartie prévue pour la construction du Stade national. Pour cet équipement, le consortium d'investisseurs chinois devait obtenir, pour une durée de 50 ans, une concession foncière de plus de 1000 ha à proximité du centre historique de Vientiane et construire un nouveau quartier urbain (New City Development Project). L'accord négocié secrètement au sommet de l'État a été remis en question en raison des rumeurs colportées à propos des contreparties en jeu - la presse locale relatait la construction d'une ville destinée à accueillir des milliers de familles chinoises. En complément, en raison du caractère fragile et sensible du site de la concession foncière choisi prenant place sur la mare du That Luang ${ }^{14}$, des ONG locales et internationales se sont mobilisées pour sensibiliser l'opinion publique. L'accord a été revu, la concession foncière a été subdivisée et de nouveaux terrains, éloignés du centre de la capitale, ont été alloués aux investisseurs étrangers (Musil et Douangmyxay, 2010).

Le fait que certaines contreparties prennent désormais la forme de concessions minières et forestières localisées à des centaines de kilomètres de Vientiane laisse à penser que les décideurs politiques qui négocient ces arrangements souhaitent soustraire les décisions prises au regard de leurs administrés et des Occidentaux, notamment des ONG, présents dans le pays. De cette manière, loin de Vientiane, le pouvoir politique est en mesure de contrôler toute contestation. Parmi les arrangements de type «équipement contre ressource naturelle », dont les contreparties sont situées dans des provinces éloignées, le cas le plus emblématique est celui de Hoang Anh Gia Lai. En l'échange de la construction du village des athlètes, l'investisseur vietnamien a obtenu une concession d'hévéa de 10000 ha dans la province d'Attapeu (Kenney-Lazar, 2010: 12-13). Cette concession s'ajoute aux projets (immobilier et infrastructure aéroportuaire) que possède déjà Hoang Anh Gia Lai dans cette province voisine du Vietnam.

Les contreparties qui apparaissent dans le cas de la construction du complexe du Stade national et dans celui du village des athlètes sont instructives. D'abord, elles illustrent clairement sur quoi reposent les modes de production contemporains des équipements dans la capitale. Ensuite, de manière plus précise, les détails apportés concernant les mécanismes de ce système d'échange montrent que les

${ }^{14}$ D'après le schéma directeur, cette mare est située en zone non constructible, notamment en raison de son rôle de bassin de rétention des eaux en période de crue du Mékong. 
opérations réalisées ne peuvent pas produire d'effet levier pour le développement urbain de Vientiane. En effet, dans la conception de ces équipements, les maîtres d'œuvre sont mandatés pour la réalisation d'un projet ponctuel. De leur côté, les autorités n'ont pas les ressources financières, et parfois humaines, nécessaires pour impulser une valorisation plus large des territoires sur lesquels sont réalisées ces opérations. Le Stade national offre là un exemple éclairant. Développé loin du centre de Vientiane, ce stade se dresse au milieu d'un paysage rural. Même si désormais des projets immobiliers et de nouvelles infrastructures (les rumeurs parlent de la construction d'un nouvel aéroport) sont prévus dans la zone du stade, ces perspectives ne sont pas garanties.

Ainsi, les seuls éléments produits à l'occasion d'évènements et apportant de la structuration physique de Vientiane sont les routes. Elles offrent des possibilités pour le développement d'opérations ponctuelles, comme notamment le projet de la Long Thanh Vientiane Economic Zone présentant une surface de 557 ha, qui sera développé au nord du pont de l'Amitié en direction de la Thaïlande le long de la route des « 450 ans ». Mais le réseau routier ne constitue qu'une trame sur laquelle des projets seront éventuellement plaqués. En matière d'urbanisme, cette logique n'est ni productive ni contreproductive, elle offre des possibilités de développement urbain qui dépendront une fois de plus d'arrangements futurs.

\section{La production urbaine au coup par coup et ses limites}

L'organisation des Jeux du Sud-Est asiatique dans la capitale laotienne en 2009 s'inscrit dans une vague d'évènements : l'accueil d'un sommet de l'Asean en 2004, les célébrations des « 450 ans » de Vientiane en 2010 et l'organisation du sommet de l'Asem (Asia-Europe Meeting) en 2012. Chaque évènement constitue une occasion pour produire de nouveaux équipements urbains (Peyronnie, 2012) et il est marqué par la démesure des investissements effectués comme par le nombre d'investisseurs étrangers impliqués. Le dernier exemple marquant fut la construction en 2012, à l'occasion du sommet de l'Asem, d'un bâtiment de grande envergure, le National Convention Center, faisant office de centre de conférences et ayant été intégralement financé par des investisseurs chinois.

Au fil des évènements organisés à Vientiane, la logique de production urbaine se veut accumulative et vise la répétition des opérations sans enclencher de processus de sédimentation. Comme nous l'avons montré à partir du cas des Jeux du Sud-Est asiatique, l'organisation d'évènements internationaux agit sur la morphologie urbaine et un effet multiplicateur apparaît dans la production des équipements urbains. Toutefois, cet effet ne favorise pas la mise en cohérence des diverses opérations d'aménagement. Une logique d'action fragmentaire émerge en lieu et place d'un projet de ville. Par conséquent, par rapport aux attentes que suscite l'urbanisme évènementiel, Vientiane constitue un cas limite. Les équipements produits ne peuvent qu'à de rares exceptions prétendre résulter de la mise en œuvre des schémas d'aménagement et participer à la structuration urbaine. 
En outre, l'analyse des opérations d'aménagement liées aux Jeux de Vientiane laisse apparaître un effet d'accoutumance des autorités laotiennes aux interventions des investisseurs étrangers. La production urbaine s'effectue au coup par coup, avec de grands projets, en mobilisant des capitaux étrangers. À chaque évènement, les autorités opèrent un tour de table des investisseurs, en particulier ceux qui disposent des meilleures connexions avec les personnes influentes du régime en place. Mais le recours aux capitaux étrangers ne s'opère pas sans contraintes. D'abord, dans bien des cas, les investisseurs imposent aux autorités laotiennes les concepteurs de projets comme les maîtres d'œuvre. Ensuite, afin de procéder aux remboursements de leurs emprunts, les autorités ont recours à des échanges particuliers: en contrepartie de la fourniture d'équipements, les investisseurs négocient et obtiennent l'exploitation de concessions foncières, forestières et minières.

Ces projets marquent le paysage urbain, mais apparaissent également comme les stigmates du dysfonctionnement de la gestion territoriale de Vientiane. Dans la capitale, l'espace urbain présente une telle plasticité qu'il n'y a rien pour créer des contraintes ou des contrepoids. De surcroît, les décideurs politiques font comme s'il n'existait aucune contrainte, en particulier environnementale et sociale, comme l'atteste le projet de quartier urbain sur la mare du That Luang. L'absence de contrepoids dans les pratiques d'aménagement à Vientiane laisse à penser que cette situation est proche du « degré zéro de l’urbanisme».

Bien que les pouvoirs publics n'aient pas lancé de politiques d'aménagement visant à résoudre des problèmes urbains à l'occasion de l'organisation des Jeux, à travers les projets routiers réalisés, une logique fondée sur le raccordement et la connexion se dégage. L'amélioration du réseau viaire permet l'apparition d'un urbanisme de détail, c'est-à-dire d'opérations qui se greffent aux axes réalisés. Bien qu'actuellement une impression de vide persiste, une intention se profile. Cela laisse supposer une évolution des dynamiques spatiales dans la capitale: si, jusqu'à présent, le développement de Vientiane s'est principalement effectué en doigts de gant et grâce à un urbanisme de projet, l'urbanisme de fragment apparaît désormais avec la constitution de zones comme celle du Stade national, et celle du campus universitaire (renforcée par la fonction d'hébergement du village des athlètes), légitimé par la création de voies d'accès. Cet urbanisme fragmentaire et diffus n'en reste pourtant qu'à ses prémices. Il présage de l'intention du régime laotien d'attirer davantage d'investissements locaux et étrangers susceptibles de lotir et dynamiser les futurs territoires urbains. 


\section{Références bibliographiques}

Andranovich, Greg, Burbank, Matthew, et Charles Heying, 2001, «Olympic cities: lessons learned from mega-event politics », Journal of urban affairs, $\mathrm{n}^{\circ} 23$, p. 113-131.

Arifin, Hadi Susilo, 2012, "Development of Landscape Architecture Education in Indonesia", Newsletter, American Society of Landscape Architects, http://www.asla.org/ppn/Article.aspx?id=34373, consulté le 23.08.13.

Banque mondiale, 2009, Lao PDR Recent Economic Developments, Vientiane, Lao PDR economic monitor - End-Year Update, The World Bank, ${ }^{\circ} 14,33$ p.

Clément Charpentier, Sophie, Clément, Pierre, Goldblum, Charles, et Christian Taillard, dir., 2010, Vientiane, architectures d'une capitale: traces, formes, structures, projets, Paris, Cahiers de l'Ipraus, $479 \mathrm{p}$.

Coaffee, Jan, 2007, «Urban regeneration and renewal », dans Gold, John, et Margaret, dir., Olympic Cities: City Agendas, Planning, and the World's Games, 1896-2012, New York et Oxon, Routledge, p. 150-162.

Creak, Simon, 2009, "Little Laos awaits its big moment», Asia Times On Line, http://www.atimes.com/atimes/Southeast_Asia/KK05Ae01.html, consulté le 23.08.2013.

Creak, Simon, 2011, "Sport as politics and history, the 25th SEA Games in Laos», Anthropology today, vol 27, ${ }^{\circ} 1$, February 2011, p. 14-19.

Gold, John, et Margaret, dir., 2007, Olympic Cities: City Agendas, Planning, and the World's Games, 1896-2012, New York et Oxon, Routledge, 348 p.

Goldblum, Charles, 2000, "Les lotissements pavillonnaires (muban chatsan) de Bangkok", dans Dureau, Françoise, et al., dir., Métropoles en mouvement. Une comparaison internationale, Paris, Anthropos/IRD, p. 60-61.

Goldblum, Charles, Franck, Manuelle, et Christian Taillard, dir., 2012, «Villes secondaires et métropolisation en Asie du Sud-Est», dans Franck, Manuelle, Goldblum, Charles, et Christian Taillard, dir., Territoires de l'urbain en Asie du Sud-Est: Métropolisations en mode mineur, Paris, Éditions du CNRS, p. 9-23.

Gratton, Chris, Shibli, Simon, et Richard Coleman, 2005, « Sport and Economic Regeneration in Cities », Urban studies, $\mathrm{n}^{\circ}$ 42, p. 985-999.

Gravari-Barbas, Maria, et Sébastien Jacquot, 2007, «L'événement, outil de légitimation de projets urbains : l'instrumentalisation des espaces et des temporalités événementiels à Lille et Gênes », Géocarrefour, vol. 82, n 3, http://geocarrefour.revues.org/2217, consulté le 24.06.2013.

Harms, Erik, 2003, «Vietnam's civilizing process and the retreat from the street: a turtle's eye view from Ho Chi Minh City », City and Society, vol. 21, n² 2, p. 182-206.

Hong, Fan, 2005, «Epilogue: Nationalism, Orientalism and Globalization: The Future of the Asian Games », Sport in Society, vol. 8, n 3, p. 515-519.

Japan International Cooperation Agency (Jica), Ministry of Public Works and Transport Lao People's democratic Republic et Katahira \& Engineers International, 2008, The study of master plan on comprehensive urban transport in Vientiane in Lao PDR, Final Report, Vientiane, Jica, 758 p.

Karnjanakit, Sombat, et Supitr Samahito, 2005, « Thailand and the Asian Games: Coping with Crisis », Sport in Society, vol. 8, n 3, p. 440-448.

Kenney-Lazar, Miles, 2010, Land Concessions, Land Tenure, and Livelihood Change: Plantation Development in Attapeu Province, Southern Laos, Vientiane, Fulbright et the National University of Laos, $47 \mathrm{p}$.

Lutan, Rusli, et Fan Hong, 2005, «The politicization of sport: Ganefo-A case study », Sport in Society, vol. 8, $\mathrm{n}^{\circ} 3$, p. 425-439.

Lutan, Rusli, 2005, «Indonesia and the Asian Games: Sport, Nationalism and the "New 
Order" », Sport in Society, vol. 8, n 3, p. 414-424.

Musil, Clément, et Sèngdara Douangmyxay, 2010, «La question foncière à Vientiane, de la politique d'ouverture économique à l'ère des projets urbains», dans Clément Charpentier, Sophie, Clément, Pierre, Goldblum, Charles, et Christian Taillard, dir., Vientiane, architectures d'une capitale : traces, formes, structures, projets, Paris, Cahiers de l'Ipraus, p. 383-402.

Peyronnie, Karine, 2012, «Des formes embryonnaires d'enregistrement de la métropolisation : Vientiane, capitale de la République démocratique populaire lao », dans Franck, Manuelle, Goldblum, Charles, et Christian Taillard, dir., Territoires de l'urbain en Asie du Sud-Est : Métropolisations en mode mineur, Paris, Éditions du CNRS, p. 75-111.

Shrestha, Omkar Lal, et Aekapol Chongvilaivan, dir., 2013, Greater Mekong Subregion, from geographical to socio-economic integration, Singapour, Iseas Publishing, $270 \mathrm{p}$.

Stuart-Fox, Martin, 2009, "Laos: The Chinese Connection», Southeast Asian Affairs, vol. 2009, p. 141-169.

Surborg, Björn, VanWynsberghe, Rob, et Elvin Wyly, 2008, « Mapping the Olympic growth machine », City, vol. 12, n³, p. 341-355. 